\title{
¿Hubo otra traducción quinientista de Celestina al italiano?
}

\author{
Amaranta Saguar García \\ Wissenschaftliche Mitarbeiterin - Universität Trier
}

\begin{abstract}
A raíz de un intercambio de ideas originado en Twitter, Lluís Batlle me hizo notar cómo cierta alusión a la traducción italiana de Celestina repetida en algunos textos de los siglos XVI y XVII no podía estar de ninguna manera remitiendo a la traducción de Alphonso Hordognez. Efectivamente, en Comparación de la lengua latina con la griega, texto que sirve de prefacio a La gramática griega escrita en lengua Castellana de Pedro Simón Abril, se alude a una traducción de Celestina al italiano en la que el traductor traslada literalmente la expresión "tomar calzas de Villadiego", dando lugar a un sinsentido traductorio (Simón Abril 1586: B1ํㅡㄹ $\left.-B 2^{\mathrm{r}}\right)^{1}$ :

Si no díganos cómo le fue al que, traduciendo a Celestina de Castellana [sic] en Toscano, para decir "que tomó calzas de Villadiego", que en nuestra lengua quiere decir "huir», dijo "que piglio caligas de Villa Iacobo», que allá quiere decir «hurtar calzas».
\end{abstract}

Sin embargo, en el pasaje correspondiente de la traducción de Hordognez, con independencia de cuál de las trece ediciones conocidas se consulte $^{2}$, la adaptación del proverbio castellano reza muy claramente «mostramo ad ogni homo li calcagni», con las inevitables variantes gráficas $^{3}$, por lo que esta traducción no puede estar en el origen de la crítica del helenista.

1.- En adelante, citamos de las obras anteriores al siglo xx desarrollando las abreviaturas sin indicación y modernizando la grafía, incluida la puntuación y el reparto de mayúsculas y minúsculas.

2.- El catálogo más completo de las ediciones de la traducción italiana de Celestina sigue siendo, a la espera de ver en qué queda la revisión iniciada por Saguar (2010, apéndice 2), el de Scoles (1964).

3.- En la primera edición conservada aparece «mostramo ad ognihomo li calcagni» (Roma 1506: $7 \mathrm{v}$ ), «mostramo ad ognihuomo li calcagni» en las ediciones inmediatamente posteriores (Milán 1514: M3v; Venecia 1515a: 13v; Milán 1515b: L5v; Milán 1519a: O1v) y, en las ediciones más tardías, bien «mostramo ad ogni homo li calcagni» (Venecia 1519b: 14v; Venecia 
Con la traducción de Hordognez descartada como fuente del ejemplo, el único otro caso conocido de adaptación quinientista de Celestina al italiano del que existen pruebas materiales, si bien en un sentido muy laxo, vendría representado por el glosario «di parecchi vocaboli Hispagnuoli difficili, contenuti quasi tutti nella Tragicomedia di Calisto e Melibea o Celestina» que Alfonso de Ulloa prepara para la edición veneciana en castellano de Celestina impresa por Gabriel Giolito y sus hermanos en 1553, y reimpresa en 1556. Sin embargo, a pesar de que en éste se traducen no pocos modismos, el de "tomar las calzas de Villadiego" no es uno de ellos, de manera que tampoco puede ser la fuente de la anécdota de Simón Abril. En consecuencia, se impone considerar la posibilidad de que el helenista se refiriera a una traducción italiana de Celestina cuyo texto no se ha conservado.

La mejor candidata es la incierta representación de Celestina durante los festejos organizados con ocasión de la boda de Lucrecia Borgia con Alfonso d'Este, que tuvieron lugar entre el 26 de diciembre de 1501 y el 6 de enero de 1502 (Alvisi 1878: 235):

Il papa, il duca, i cardinale diedero rappresentazioni in onori degli ospiti —quelle egloghe o pastorali che allora alla corte di Spagna erano in voga - migliore di tutte la Celestina di Rodrigo da Cota che nel 1505 tradotta in italiano fu dedicata ad una nipote di Giulio II; le allusioni agli sponsali, ai nomi di Alessandro, di Cesare, di Ercole le rendevano più accette.

Sin embargo, dejando a un lado las inexactitudes sobre el autor del original y la dedicatoria de la traducción, que ya hicieran dudar a Emma Scoles (1961: 158-159 n2) sobre la credibilidad de este pasaje, nada indica que la representación se hiciera en italiano, a pesar de la certeza con que lo afirma Juan Carlos de Miguel y Canuto (2003: 73). De hecho, la referencia a la traducción de Hordognez como novedad respecto a las obras representadas, así como la alusión a la moda de la corte española y la ascendencia valenciana de la familia Borgia, hacen pensar que, de haberse representado realmente Celestina, lo habría sido antes en su lengua original que en italiano, por lo que tampoco puede atribuirse a esta representación la anécdota de Simón Abril.

La consulta de los inestimables trabajos de Joseph T. Snow (1997; 2001; 2002; 2013) sobre la recepción de Celestina no nos ha puesto tras la pista de ninguna otra potencial traducción quinientista de Celestina, sin embargo, sí hemos encontrado más alusiones a la traducción defectuosa de «tomar las calzas de Villadiego» en varios textos del siglo XVII, tanto cas-

1525a: K6v; Venecia 1525b: K6v; Venecia 1543: K6r; Venecia 1531a: K6v), bien «mostramo ad ogni huomo li calcagni» (Venecia 1525c: K6v; [Venecia] 1531b: K1v; [Venecia] 1535: K1v; [Venecia] 1541: K1v). 
tellanos como italianos, donde se utiliza para ejemplificar las dificultades de la traducción. Así en la Reducción de las letras y arte para enseñar a hablar los mudos de Juan Pablo Bonet, que remite explícitamente a Simón Abril (Bonet 1620: 290-291):

Y a este mismo propósito trae Simón Abril el rigor de la traducción que hizo el que tradujo el libro de Celestina en Italiano, que por el frasis tan usado de "Tomó las calzas de Villadiego» dijo "Piglio le calce di Villa Iacobo», que nosotros queremos decir "Huyóse», y él entendió que hurtó las calzas a uno que se llamaba Villa Diego.

En el Tesoro de la lengua castellana (Covarrubias 1611: 506r):

INTÉRPRETE: el que vuelve las palabras y conceptos de una lengua en otra, en el cual se requiere fidelidad, prudencia y sagacidad, y tener igual noticia de ambas lenguas, y lo que en ellas se dice por alusiones y términos metafóricos mirar lo que en estotra lengua lengua le puede corresponder, como notan el descuido del que volvió la Celestina, que por la cláusula «Tomó las calzas de Villa Diego» dijo simplemente «Piglio le calce de Villa Iacome», habiendo de atender al sentido que era echó a huir.

O en Il compendio del signor Massimo Troiano tratto dalle "Osservationi della lingua castigliana» del signor Giovanni Miranda (Troiano 1601: 205):

E sarebbe appunto il traslatamento di quel proverbio della bellissima Tragicommedia di Celestina, dove traslatando uno "Tomò las calças de Villadiego» (che e tanto in Ispagnuolo come dir «Marciò via: Se ne fuggì») disse "Presse le calze de Villa Iacopo", perciocchè "Diego», in Ispagnuolo, vuol dire "Iacopo», in Italiano.

Su valor como ejemplo de las dificultades traductorias se prolonga incluso hasta el siglo XIX (Castro 1848: 194):

La mayor parte de los refranes españoles no pueden ser fielmente traducidos a los idiomas extraños, porque en ellos nada significarían. Uno de los traductores que en Italia tuvo la tragicomedia de Celestina, leyendo en ella que uno había tomado las calzas de Villadiego, que en castellano significa "huir», interpretó del modo siguiente: «Piglio le calce de Villa Iacobo», entendiendo que uno de los interlocutores había hurtado las calzas a un hombre que se llamaba Villa Diego. 
Pero una comparación rápida de los cinco ejemplos pone de manifiesto que la formulación de la traducción errónea no coincide excepto entre el texto de Bonet y el de Castro; tan similares que no nos parece arriesgado afirmar que el primero sirvió de fuente al segundo ${ }^{4}$ :

\section{[...] dijo «que piglio caligas de Villa Iacobo» (Simón Abril 1586: $\mathrm{B} 1^{\mathrm{v}}-\mathrm{B} 2^{\mathrm{r}}$ ) \\ [...] disse «Presse le calze de Villa Iacopo» (Troiano 1601: 205) \\ [...] dijo simplemente «Piglio le calce de Villa Iacome» (Covarrubias 1611: 506 ${ }^{\text {) }}$ \\ [...] dijo «Piglio le calce di Villa Iacobo» (Bonet 1620: 291) \\ [...] interpretó del modo siguiente: «Piglio le calce de Vi- lla Iacobo» (Castro 1848: 194)}

Esta falta de correspondencia generalizada apunta a que no existió una fuente textual a la que se remontaran todos los autores. Es decir, las diferencias redaccionales entre los testimonios de esta anécdota son demasiado notables como para poder tratarse de meras variantes de transmisión de la traducción italiana de Celestina a la que remite, por lo que algunos de los autores tienen que haberse hecho eco de ésta necesariamente de manera indirecta, sin haber visto con sus propios ojos la supuesta traducción italiana de Celestina a la que se le atribuye la traducción defectuosa de "tomar las calzas de Villadiego».

En el caso de Bonet no hace falta demostrar que Simón Abril es su fuente, por más que no lo cite literalmente, ya que así lo reconoce. Asimismo, las evidentes deudas de Castro con Bonet hacen de Simón Abril también su término último de referencia. ¿Y en cuanto al propio Simón Abril? Dado que no hallamos ninguna evidencia textual de la existencia de la traducción a la que remite, su anécdota adquiere todo el color de una historieta similar al las recogidas, por ejemplo, en la Floresta española de Melchor de Santa Cruz (1574): circunstanciales, muchas de ellas anónimas y donde la historicidad no importa tanto como la comicidad del relato. Así pues, aunque nada impide que existiera un personaje histórico real que tradujera literalmente la expresión «tomar las calzas de Villadiego» al verter Celestina al italiano, ni que su metedura de pata hubiera pasado de boca en boca hasta llegar a los oídos de Simón Abril, Troiano y Covarrubias, tampoco existe nada en contra de que la anécdota fuera absolutamente falsa e inventada, popularizada por su componente humorístico. Incluso puede perfectamente tratarse de una historieta creada ad hoc por el propio

4.- No cabe duda de que Castro leyó el texto de Bonet, pues lo cita explícitamente: «No dejó escrito arte, pero su falta fue suplida luego por Juan Pablo Bonet con la publicación de un precioso tratado que corre impreso con el siguiente título: Reducción de las letras y arte para enseñar a hablar los mudos (Madrid, 1620)» (Castro 1848: 53). 
Simón Abril para acompañar su reflexión sobre las dificultades de la traducción y luego popularizada, en tanto no encontramos referencias a la pifia traductoria anteriores a la publicación de su Comparación de la lengua latina con la griega en 1586, de la que Troiano y Covarrubias se habrían hecho eco indirectamente; de ahí las diferencias textuales.

No obstante lo anterior, la prueba más determinante de la ficcionalidad de dicha anécdota y, por lo tanto, de la inexistencia de una traducción quinientista de Celestina desconocida reside en la propia formulación del texto pues, en la escena pertinente del auto duodécimo, no es «tomó las calzas de Villadiego» lo que leemos, sino "Apercíbete, a la primera vez que oyeres, tomar calzas de Villadiego» (Rojas y antiguo autor 2011: 242). En consecuencia, la traducción defectuosa no puede relacionarse con el texto celestinesco y la historieta no puede ser real, sino únicamente inventada, ya sea por Simón Abril o por otro. Es decir, nunca existió otra traducción quinientista de Celestina al italiano distinta de la de Hordognez; al menos no una que excediera el ámbito de lo estrictamente personal y privado.

\section{Bibliografía}

AlvisI, Edoardo (1878), Cesare Borgia, duca di Romagna, Imola, Tipografia d'Ignazio Galeati e figlio.

BONET, Juan Pablo (1620), Reduction de las letras y arte para enseñar a ablar los mudos, Madrid, Francisco Abarca de Angulo. Disponible on-line $<$ https://books.google.de/books?id=yp9XAAAAcAAJ>.

Castro, Adolfo de (ed.), (1848), El buscapié, Cádiz, Revista Médica. Disponible on-line <https://books.google.de/books?id=vMOH1F1YG_MC>.

CovarRubias, Sebastián de (1611), Tesoro de la lengua castellana, o española, Madrid, Luis Sánchez. Disponible on-line <https://books.google.de/ books?id=qKm8nzelynUC $>$.

Hordognez, Alphonso (1506), Tragicocomedia di Calisto e Melibea, Roma, Eucario Silber. Disponible on-line $<$ http://bdh-rd.bne.es/viewer. vm?id $=0000011584$ \& page $=1>$.

- (1514), Tragicocomedia di Calisto e Melibea, Milán, Zanotto da Castione. Disponible on-line <http://bdh-rd.bne.es/viewer.vm?id=0000012203 \&page $=1>$.

- (1515a), Tragicocomedia di Calisto e Melibea, Venecia, S.i. Disponible online <http://gallica.bnf.fr/ark:/12148/bpt6k855277w>.

- (1515b), Tragicocomedia di Calisto e Melibea, Milán, Vicentio Minutiano. Disponible on-line <http://www.opal.unito.it/psixsite/Teatro\%20 italiano \%20del\%20XVI\%20e\%20XVII\%20secolo/Elenco\%20opere/ image78.pdf>. 
- (1519a), Tragicocomedia di Calisto e Melibea, Milán, Giovann'Angelo Scinzenzeler.

- (1519b), Tragicocomedia di Calisto e Melibea, Venecia, Cesare Arrivabene. Disponible on-line <http://www.opal.unito.it/psixsite/Teatro\%20 italiano\%20del\%20XVI\%20e\%20XVII\%20secolo/Elenco\%20opere/ image77.pdf>.

- (1525a), Tragicocomedia di Calisto e Melibea., Venecia, Francesco Caron. Disponible on-line <http://books.google.de/books?vid=BNC: 1001968120>.

- (1525b), Tragicocomedia di Calisto e Melibea, Venecia, Gregorio de Gregorii. Disponible on-line <http://www.opal.unito.it/psixsite/Teatro $\% 20$ italiano \%20del $\% 20 X V I \% 20 \mathrm{e} \% 20 X V I I \% 20$ secolo/Elenco\%20opere/ image29.pdf $>$.

- (1525c), Tragicocomedia di Calisto e Melibea, Venecia, Francesco Caron. Disponible on-line <http://www.opal.unito.it/psixsite/Teatro $\% 20$ italiano\%20del\%20XVI\%20e\%20XVII\%20secolo/Elenco\%20opere/ image79.pdf $>$.

- (1531a), Tragicocomedia di Calisto e Melibea, Venecia, Bindoni y Passini. Disponible on-line <http://gallica.bnf.fr/ark:/12148/bpt6k855276h>.

- (1531b), Tragicocomedia di Calisto e Melibea, [Venecia], Marchio Sessa. Disponible on-line <http://gallica.bnf.fr/ark:/12148/bpt6k8552754>.

- (1535), Tragicocomedia di Calisto e Melibea, [Venecia], Pietro de Nicolini da Sabio. Disponible on-line <http://www.urfm.braidense.it/ $\mathrm{rd} / 01978 . \mathrm{pdf}>$.

- (1541), Tragicocomedia di Calisto e Melibea, [Venecia], G.A. y P. de Nicolini da Sabio. Disponible on-line <http://bdh-rd.bne.es/viewer. vm?id $=0000077245 \&$ page $=1>$.

- (1543), Tragicocomedia di Calisto e Melibea, Venecia, Bernardino de Bendoni.

Miguel y Canuto, Juan-Carlos de (2003), "Sosta nel labirinto: bilancio bibliografico sulla prima traduzione italiana di La Celestina», Studi e problemi di critica testuale $\mathrm{n}^{\circ}$ 67, pp. 71-108.

RojAs, Fernando de (1553), Tragicomedia de Calisto y Melibea, Venecia, Gabriel Giolito.

- (1556), Tragicomedia de Calisto y Melibea, Venecia, Gabriel Giolito. Disponible on-line <http://www.mdz-nbn-resolving.de/urn/resolver. pl?urn=urn:nbn:de:bvb:12-bsb10189656-6>.

RoJAS, Fernando de, y "antiguo autor» (2011), La Celestina: tragicomedia de Calisto y Melibea, ed. Francisco Lobera et al., Madrid, Real Academia Española.

SAguAR, Amaranta (2010), "The Textual Filiation of the Early Editions of Hordognez' Tragicomedia de Calisto y Melibea (with some notes on the German Translation of Celestina)", Extended Essay, University of Oxford. 
SCOLES, Emma (1961), "Note sulla prima traduzione italiana della Celestina», Studj Romanzi n 33, pp. 153-217.

- (1964), «La prima traduzione italiana della Celestina: repertorio bibliografico", Studi di letteratura spagnola, pp. 209-230.

Simón ABRIL, Pedro (1586), La gramatica griega escrita en lengua Castellana: para que desde luego puedan los niños aprender la lengua griega juntamente con la latina conforme al consejo de Quintiliano con el aiuda i fauor de la vulgar, Zaragoza, Lorenzo y Diego de Robles. Disponible on-line $<$ http:// bvpb.mcu.es/es/consulta/registro.cmd?id=400903 >.

Snow, Joseph (1997), "Hacia una historia de la recepción de Celestina: 1499-1822», Celestinesca n 21.1-2, pp. 115-173.

- (2001), «Historia de la recepción de Celestina: 1499-1822. II (14991600)", Celestinesca n 25.1-2, pp. 199-282.

- (2002), «Historia de la recepción de Celestina: 1499-1822. III (16011800)», Celestinesca n 26.1-2, pp. 53-121.

- (2013), «Historia crítica de la recepción de Celestina: 1499-1822. Entrega IV.», Celestinesca n० 37, pp. 151-206.

Troiano, Massimo (1601), Il compendio del signor Massimo Troiano tratto dalle "Osseruationi della lingua castigliana" del signor Giouanni Miranda, Firenze, Bartolommeo Sermartelli il giouane. Disponible on-line $<$ https://books.google.de/books?id=G7dknj_E2dYC $>$. 
Saguar García, Amaranta, «¿Hubo una traducción quinientista de Celestina al italiano?», Celestinesca 39 (2015), pp. 53-60.

\section{RESUMEN}

Algunos textos españoles e italianos de los siglos XVI y XVII parecen aludir a una traducción quinientista de Celestina al italiano diferente de la de Alphonso Hordognez. Este artículo analiza varias referencias a dicha traducción para intentar determinar si existió realmente o si se trata de una traducción fantasma.

Palabras Clave: Traducción de Celestina, Celestina en italiano.

\section{ABSTRACT}

Some texts of the sixteenth and the seventeenth centuries seem to make reference to a sixteenth-century translation of Celestina into Italian other than Alphonso Hordognez's. In this article, the author analyses some references to the abovementioned translation in order to determine if it really existed or not.

KEY WORDS: Celestina in translation, Celestina in Italian.

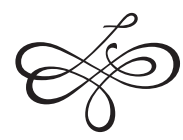

